\title{
Adnexal Torsion in Pregnancy: Should We Conserve or Not?
}

\author{
K. Harou, K. Elbehja, M. Nafidi, H. Asmouki, A. Soummani \\ Obstetrics and Gynecology Department, Mohammed VI University Hospital Center, Faculty of Medicine and Pharmacy, \\ Marrakech, Morocco \\ Email: meriem.nafidi@gmail.com
}

How to cite this paper: Harou, K., Elbehja, K., Nafidi, M., Asmouki, H. and Soummani, A. (2021) Adnexal Torsion in Pregnancy: Should We Conserve or Not? Open Access Library Journal, 8: e7476.

https://doi.org/10.4236/oalib.1107476

Received: April 30, 2021

Accepted: June 20, 2021

Published: June 23, 2021

Copyright $\odot 2021$ by author(s) and Open Access Library Inc.

This work is licensed under the Creative Commons Attribution International License (CC BY 4.0).

http://creativecommons.org/licenses/by/4.0/

\section{(c) (i) Open Access}

\begin{abstract}
Adnexal torsion is a rare complication estimated to occur in approximately $1 / 1000$ pregnancies and considered as a surgical emergency. The clinical diagnosis remains difficult given the non-specificity of the clinical picture. Since the functional cysts during pregnancy often resolve spontaneously, the surgeon may opt for conservative management by de-torsion by laparoscopy. The adverse infant-related outcomes, even weakly described over the years, impose considerable harmlessness with respect to progressive pregnancy. In this report, we will describe the management of a torsion of an ovarian cyst in 11 weeks of pregnancy, and show that detorsion should be the procedure of choice for cases of simple ovarian torsion.
\end{abstract}

\section{Subject Areas}

Gynecology \& Obstetrics

\section{Keywords}

Torsion, Pregnancy, Laparoscopy, Conservative Treatment

\section{Introduction}

Adnexal torsion is a rare cause of acute abdominal pain during pregnancy [1]. It is frequently associated with ovarian stimulation for in vitro fertilization (IVF) or with ovarian masses, mainly of functional origin.

The clinical symptoms are non-specific and could be confused with other acute abdominal conditions, such as acute appendicitis, renal colic, and cholecystitis.

It is a surgical emergency which requires a quick diagnosis and an immediate management. Early diagnosis is essential to preserve organ function and fertility. 
Traditionally, abdominal complications during pregnancy have been treated by means of laparotomy.

Nowadays, laparoscopy is considered the preferable surgical option until approximately the 16th week of gestation [2]. The big dilemma in this association is diagnosis difficulty and the decision of an optimal therapeutic management.

We report here the case of torsion of an ovarian cyst in a pregnant woman carrying a pregnancy of 11 weeks of amenorrhea.

\section{Case Report}

The patient was a 40-year-old pregnant woman, with two children by caesarean section, who presented to our center for acute onset of stabbing and non-radiating continuous lower right abdominal pain, of 4 hours duration. She had no history of prior abdominal trauma.

The patient experienced sudden onset of severe progressive abdominal pain. She could not obtain relief form the pain after changing position, and it was accompanied by nausea, vomiting. Physical examination revealed that the lower abdominal tenderness was worse on the right side, and there were no signs of peritonitis.

Transvaginal ultrasound indicated an enlarged right ovary with multiloculated cyst measuring $13.1 \times 9.4 \mathrm{~cm}$ with sparse blood flow (Figure 1 ); the left ovary appeared normal with an evolving monofoetal pregnancy of 11 weeks (Figure 2).

Laboratory blood examination revealed increased white blood cell count $(10,140$ cells/ $\mu \mathrm{l})$, neutrophil granulocyte percentage $(92 \%)$.

In an emergency context and given the obstetric history of the patient, we opted for surgical treatment by Pfannenstiel incision. It was performed 1hour later, which revealed that the right ovary was cystic and multilocular with a black purplish surface and thin wall. The right ovary and right fallopian tube had undergone $960^{\circ}$ torsion (4 rotations) (Figure 3). The cyst in the right ovary was accidently ruptured during the fourth detorsion and light-yellow fluid was seen during the operation. Decorpulation of the residual hematoma in the ovarian shell was realized and despite the initial ischemic appearance of the ovary, a sign of ovarian vitality has appeared a few minutes later so we opted for conservative treatment.

The patient benefited from a preventive progestogen tocolysis postoperatively. 1 week after surgery, the patient presented normal ovarian size on both sides and progressing pregnancy without incident. She still benefits from her pregnancy follow-up in our center with cesarean section scheduled at 39 week

\section{Discussion}

Adnexal torsion is a maternal morbidity of pregnancy that demands emergent surgery and exposes the gravida and fetus to increased risk. It accounts for approximately $3 \%$ of gynecological emergencies and $10 \%-20 \%$ of ovarian torsions occur during pregnancy [3]. 


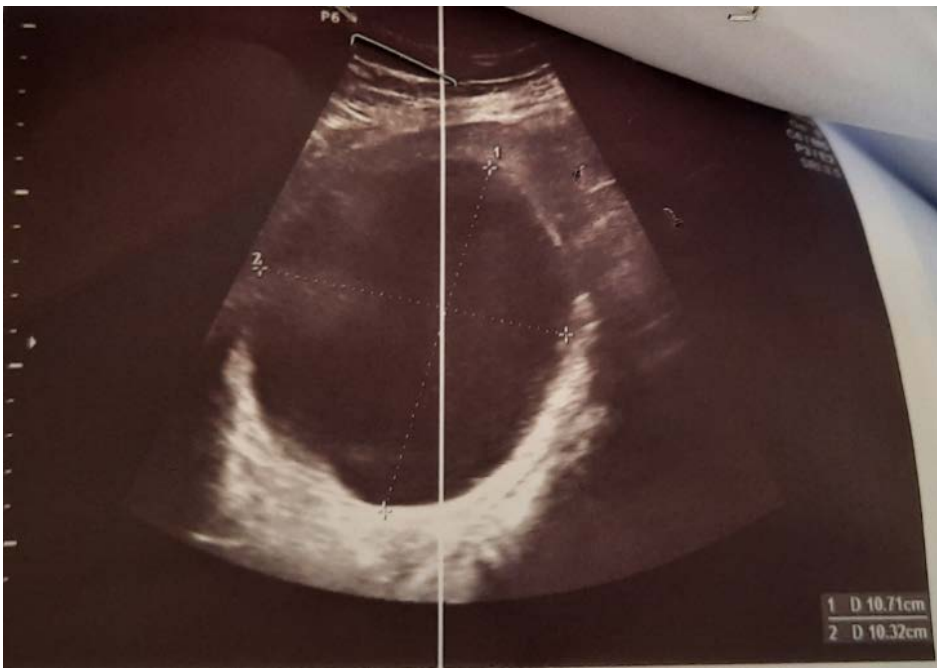

Figure 1. Multiloculated cyst of the right ovary with evolving pregnancy.

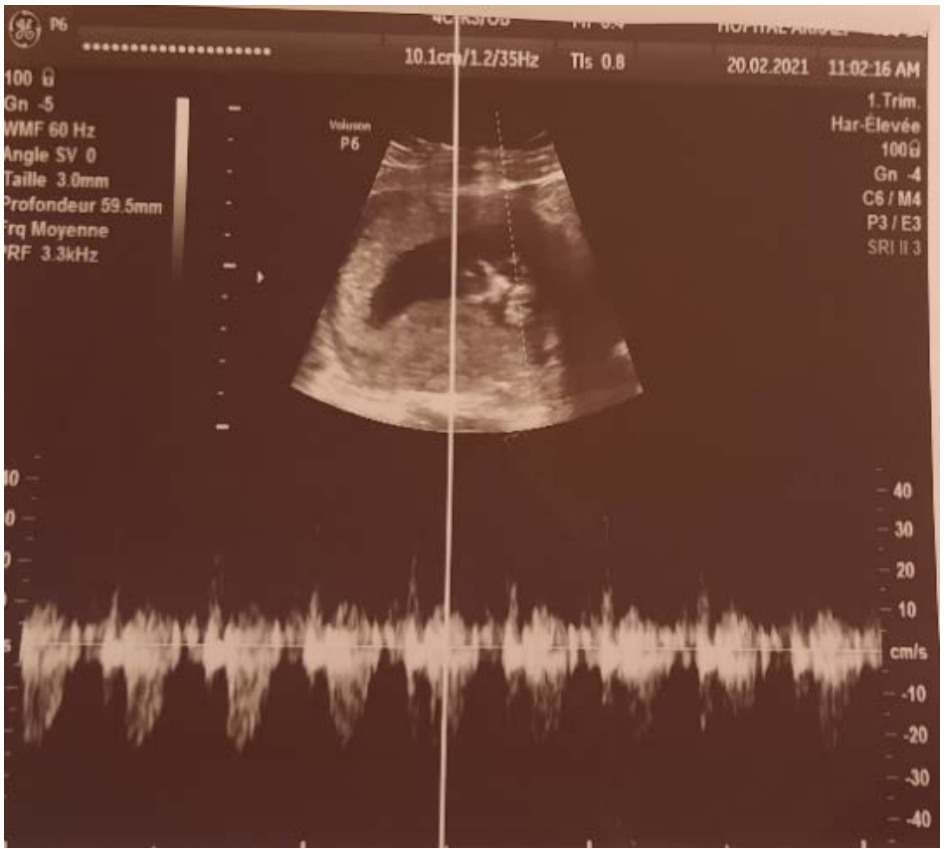

Figure 2. Evolving monofoetal pregnancy.
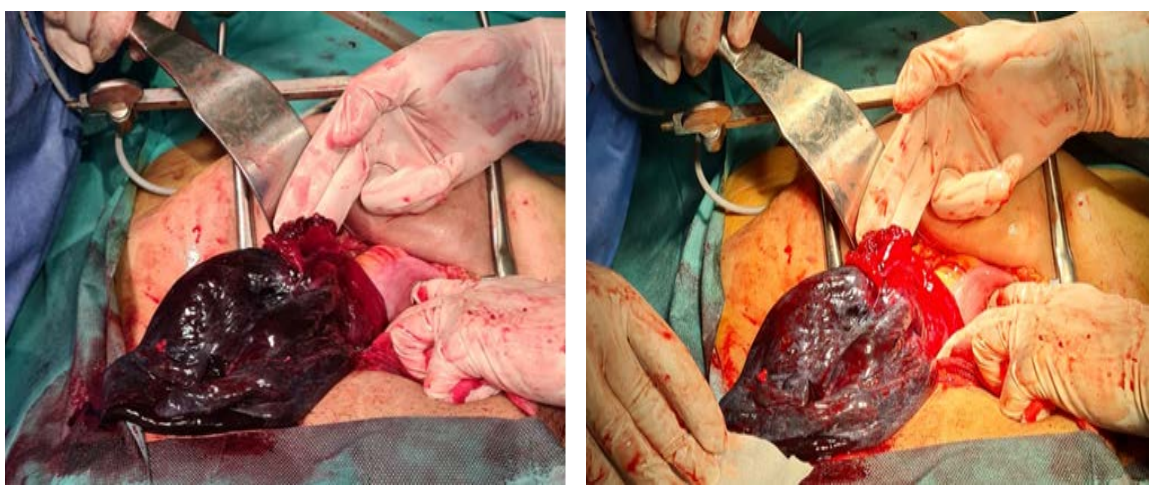

Figure 3. Torsion and detorsion of the right ovarian cyst. 
Adnexal torsion usually presents in the first trimester, when the uterus is moving out of the pelvis, although some cases have been described in the second and, rarely, in the third trimester. Most of the described torsions have been in the right adnexa, probably because the sigmoid limits the mobility of the left ovary.

The first trimester torsion is usually associated with functional ovarian cysts and the gradual regression of these ovarian cysts during the second and third trimester is connected to a lower risk of torsion [4]. The commonest type of ovarian tumors encountered in pregnancy is cystic teratoma, paraovarian cyst, serous cystadenoma, corpus luteal cysts [5] [6].

Complications of the cysts associated with pregnancy are torsion of the cyst, rupture, infection, malignancy, impaction of cyst in pelvis causing retention of urine, obstructed labour and difficult presentations of the fetus.

The clinical exam is often poor and not relevant. The clinical diagnosis is not specific and the main symptom is pelvic pain. Differential diagnosis includes uterine leiomyoma's, non-pregnant horn of bicornuate uterus, appendicle abscess, diverticular abscess, pelvic kidney, retroperitoneal tumors, ectopic pregnancy and retroverted gravid uterus. The preoperative diagnosis is difficult. Torsion of the ovarian pedicle results in circulatory stasis that is initially venous, but becomes arterial as the torsion and the resultant edema progress. When complete torsion with hemorrhagic necrosis is suspected, immediate surgery is necessary. If there is a delay in the diagnosis and the torsion persists for more than 36 - $48 \mathrm{~h}$, the lesions of the ovary are irreversible and a conservative, organ-preserving approach is not possible. However, it has been described that viable ovarian tissue can be detected even after 48 h of torsion. Conclusively, ovarian torsion often remains a diagnostic dilemma, largely because of the non-specific clinical, laboratory and imaging findings.

Ultrasound findings, including solid, cystic and complex pelvic tumors, with or without fluid collections in the pouch of Douglas, are non-specific for the diagnosis of adnexal torsion. Color Doppler sonography has been proved useful and the main sign is the absence of intraparenchymal ovarian blood flow. The disappearance of ovarian flow depends on the stage of torsion, therefore close monitoring is necessary in order to achieve timely management with a conservative approach.

The Doppler flow can persist in spite the vascular impairment: false negative MRI can be also applied in pregnant women without the hazards associated with ionizing radiation. MRI findings in adnexal torsion include a thick edematous pedicle and ovary, lack of enhancement and signal intensities indicative of hemorrhage. MRI can be useful in case of solid mass and suspected malignancy

The management of adnexal torsion on cyst in pregnancy is balanced between maternal and fetal complications. Surgical management is the reference ,requiring a good knowledge of laparoscopic surgery and the management of prenatal anesthesia [7]. Emergency surgery should be done as soon as possible, for pa- 
tients with acute abdominal pain with a high suspicion of ovarian torsion ,suspected pelviperitonitis or no response to symptomatic treatment, otherwise the decision is very difficult. Once the surgical decision is taken, The difficulty of the surgical approach is added to decide to be conservative or not in case of necrosis. We usually decide not to conserve in case of irreversible ischemia but several studies advise to be conservative despite the color of the ovary. Some studies have proven that it is not accurate to determine the activity of the ovary based on the color during the surgery [8] [9]. Although the appearance of the ovaries was found to be severely ischemic during the operation, all underwent detorsion with or without evacuation of the hematoma. Follow-up sonography showed all ovaries had follicular development except one in this study.

Adnexal torsion on cyst necessitates prompt surgical intervention, detorsion and cystectomy with preserving ovarian function are the main treatment methods. Recently, detorsion via laparoscopy has become nearly standard treatment [10].

Cases of adnexal torsion occurring during the first trimester of pregnancy should preferably undergo laparoscopy, which is suitable for diagnosis, evaluation and treatment. Furthermore, the possibility of recurrence, and pregnancy outcomes for the patient should be considered .The oophoropexy is strongly recommended to avoid recurrent torsion; it can also be prevented by shortening the utero-ovarian ligament [11].

With our patient, the treatment carried out was detorsion and cystectomy with preserving the ovary. We carefully considered the anesthesia-related drugs for the foetus, which can be used during pregnancy. Progesterone was administered to the patient who underwent surgery in the first trimester in our case, According to the guidelines of the Society of American Gastrointestinal and Endoscopic Surgeons [10].

\section{Conclusions}

In conclusion, adnexal torsion is a rare event during pregnancy, which requires differential diagnosis from other diseases presenting with abdominal pain. It necessitates a prompt surgical intervention, because any delay leads to irreversible ovarian necrosis. The occurrence of complications requires surgery in order to avoid the ovarian damage.

The aim of our publication is to show that conservative treatment as detorsion should always be the first and preferable treatment in cases of ovarian torsion, irrespective of grade of necrosis at exploration.

Despite the technological advances in ultrasonography, the diagnosis of the disease is difficult, especially during pregnancy and occasionally remains a diagnostic and management dilemma.

\section{Acknowledgements}

We would like to thank the patient for giving us permission to publish this report. 


\section{Conflict of Interest Statement}

The author(s) declared no potential conflicts of interest with respect to the research, authorship, and/or publication of this article.

\section{References}

[1] Condous, G., Khalid, A., Okaro, E. and Bourne, T. (2004) Should We Be Examining the Ovaries in Pregnancy? Prevalence and Natural History of Adnexal Pathology Detected at First-Trimester Sonography. Ultrasound in Obstetrics \& Gynecology, 24, 62-66. https://doi.org/10.1002/uog.1083

[2] Mashiach, S., Bider, D., Moran, O., Goldenberg, M. and Ben-Rafael, Z. (1990) Adnexal Torsion of Hyperstimulated Ovaries in Pregnancies after Gonadotropin Therapy. Fertility and Sterility, 53, 76-80. https://doi.org/10.1016/S0015-0282(16)53219-1

[3] Aggarwal, P. and Kehoe, S. (2011) Ovarian Tumours in Pregnancy: A Literature Review. European Journal of Obstetrics \& Gynecology and Reproductive Biology, 155, 119-124. https://doi.org/10.1016/j.ejogrb.2010.11.023

[4] Melcer, Y., Dvash, S., Maymon, R., Pekar-Zlotin, M., Vaknin, Z., Tzur, T., et al. (2021) Torsion of Functional Adnexal Cysts in Pregnancy: Aspiration and Drainage are Important in Preventing Recurrence. The Israel Medical Association Journal, 23, 48-51.

[5] Smorgick, N., Pansky, M., Feingold, M., Herman, A., Halperin, R. and Maymon, R. (2009) The Clinical Characteristics and Sonographic Findings of Maternal Ovarian Torsion in Pregnancy. Fertility and Sterility, 92, 1983-1987.

https://doi.org/10.1016/j.fertnstert.2008.09.028

[6] Ginath, S., Shalev, A., Keidar, R., Kerner, R., Condrea, A., Golan, A., et al. (2012) Differences between Adnexal Torsion in Pregnant and Nonpregnant Women. Journal of Minimally Invasive Gynecology, 19, 708-714. https://doi.org/10.1016/j.jmig.2012.07.007

[7] Schmeler, K.M., Mayo-Smith, W.W., Peipert, J.F., Weitzen, S., Manuel, M.D. and Gordinier, M.E. (2005) Adnexal Masses in Pregnancy: Surgery Compared with Observation. Obstetrics \& Gynecology, 105, 1098-1103. https://doi.org/10.1097/01.AOG.0000157465.99639.e5

[8] Parelkar, S.V., Mundada, D., Sanghvi, B.V., Joshi, P.B., Oak, S.N., Kapadnis, S.P., et al. (2014) Should the Ovary Always Be Conserved in Torsion? A Tertiary Care Institute Experience. Journal of Pediatric Surgery, 49, 465-468. https://doi.org/10.1016/j.jpedsurg.2013.11.055

[9] Kives, S., Gascon, S., Dubuc, É. And Van Eyk, N. (2017) No. 341-Diagnosis and Management of Adnexal Torsion in Children, Adolescents, and Adults. Journal of Obstetrics and Gynaecology Canada, 39, 82-90.

https://doi.org/10.1016/j.jogc.2016.10.001

[10] Pearl, J., Price, R., Richardson, W. and Fanelli, R. (2011) Guidelines for Diagnosis, Treatment, and Use of Laparoscopy for Surgical Problems during Pregnancy. Surgical Endoscopy, 25, 3479-3492. https://doi.org/10.1007/s00464-011-1927-3

[11] Obut, M. and Değer, U. (2019) A New Technique of Oophoropexy: Folding and Fixating of Utero-Ovarian Ligament to Round Ligament in a Patient with Reccurrent Ovarian Torsion. Case Report Obstetrics Gynecology, 2019, Article ID: 7647091. https://doi.org/10.1007/s00464-011-1927-3 